\title{
Design of Small Excavator Health Management System
}

\author{
Xia Yimin ${ }^{1}$, Liu Shang ${ }^{2+}$, Yuan Ye ${ }^{3}$, Shi Yupeng ${ }^{1}$ and Fu Jie ${ }^{1}$ \\ ${ }^{1}$ College of Mechanical and Electrical Engineering, Central South University, ChangSha; 410083, China \\ ${ }^{2}$ School of Software, Central South University, ChangSha; 410083, China \\ ${ }^{3}$ Zoomlion Heavy Industry Science and Technology Co., Ltd.; 410013, China
}

\begin{abstract}
In view of the current problems in the working process of small excavators. Such as various health indicators cannot be handled in a centralized manner, conditions cannot be identified in time and energy consumption cannot guide the main engine's energy-saving work. Based on demand analysis and functional modules, a small excavator health management system was established. Build a software system based on a three-tier browser/server architecture. Writing background code through C\# language to complete the database connection and operation. Establish models such as energy consumption analysis and condition identification to evaluate the health indicators such as fuel consumption and operating conditions of the main engine. Visualize the results of the assessment through curves, icons, reports, and more.
\end{abstract}

Keywords: energy analysis, condition identification, management system, small excavator.

\section{Introduction}

A small hydraulic excavator is a versatile engineering machine that can be widely used in hydraulic engineering, transportation, power engineering, mining and other engineering construction[1]. As people continue to improve the excavator. On the one hand, the energy consumption of the excavator has become a factor of competition among major manufacturers. On the other hand, new requirements have been put forward for the diagnosis of excavator failures. In recent years, there have also been some technological innovations and practical achievements in these areas. Such as the life cycle health management system for full-face roadheaders [2]. The comprehensive spacecraft health management system [3] and the analysis and management of Acrel-5000 building energy consumption [4]. Internet of things-based equipment health management system [5] and so on. However, most of these software are based on fault prediction and health management (PHM) and individual energy consumption analysis. Only a small number of systems involve multi-functional management. There is no research and development for systems that can realize condition identification, energy consumption analysis and fault diagnosis.

The core of health management is the use of advanced sensor technology integration. The use of various algorithms and intelligent models to complete the system status monitoring, fault diagnosis / prediction[6]. Then make appropriate decisions on maintenance activities based on diagnostic or predictive information to increase system utilization. Therefore, the trade-off between safety and economy in use and maintenance can be reasonably weighed to ensure the lowest cost in the whole life cycle[7]. From the small excavator condition identification, energy analysis, fault diagnosis for all-round management, the main research content of this topic. The purpose of the small excavator health management system is to convert it into useful health management information through relevant work data of small excavators.

Based on the above analysis, this paper will study and design a small excavator health management system based on ASP.NET technology. First of all, according to ASP.NET's architecture design, the system

\footnotetext{
+ Corresponding author. Tel.: 13278887787

E-mail address: 1shang@csu.edu.cn.
} 
is divided into three layers: the presentation layer, business logic layer and data access layer, and the system framework is built. Secondly, based on this, the identification of working conditions, energy consumption analysis, and fault diagnosis are three. In terms of designing a small excavator health management system that meets the requirements.

\section{System Architecture}

Taking into account the business process of the enterprise and the convenience of system maintenance, the system is developed using a three-tier Browser/Server architecture. Fig. 1 shows the three-tier architecture. Fig. 2 shows the architecture of the management system, which consists of DAL (Data Access Layer), BLL (Business Logic Layer), and UI (Representation Layer). In a three-tier architecture, it is used only for data storage, except that it stores complex data. DBUtility is a method of connecting to a database. It contains generic code that accesses the database and is called by the data access class in the DAL. It is not necessary. This method can make the connection more convenient and faster using this method.

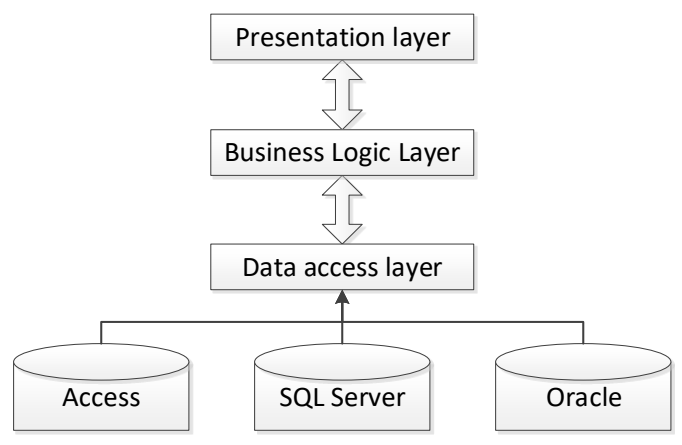

Fig. 1: Three-tier architecture diagram.

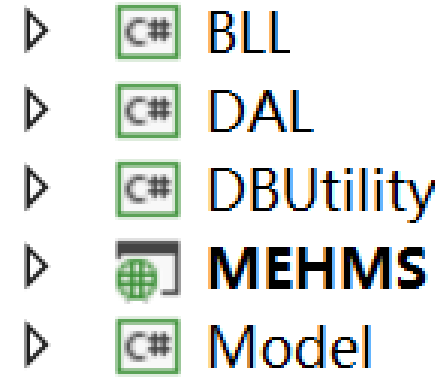

Fig. 2: Health management system three-tier architecture.

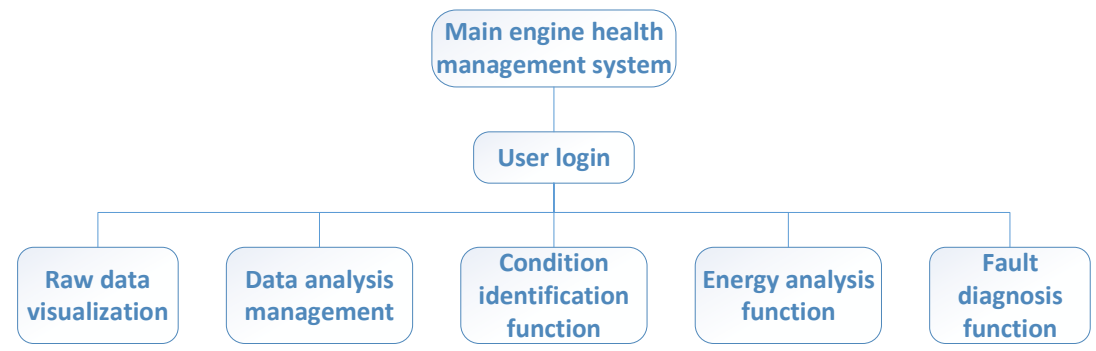

Fig. 3: Function structure of health management system.

\section{System Design}

\subsection{Overall System Design}

Through researching the health problems related to small excavators and consulting relevant documents at home and abroad, this paper has designed the main functions and modules of the small excavator health management system. It not only needs to meet the management personnel's operational recommendations, navigation is clear, but also needs to meet management personnel. Fault diagnosis, usage information and related data statistics and other functions. At present, the tasks that the system needs to achieve mainly include: user login (authentication of user information, security of data security), visualization of raw data (visualization of data flowing into the database, including curve display of each channel, contrast display of the same channel under different experiments ), data analysis and management (pre-processing of acquired data, application of collected data, simple logic judgment or numerical calculation), condition identification module (recognition of main engine operating conditions), energy consumption analysis module ( Through the calculation of raw data and algorithms, the corresponding energy consumption indicators are drawn and graphed), fault diagnosis modules (acquisition of data, realization of diagnosis). The overall function structure of the tool management information system is shown in Fig. 3.

\subsection{System Database Design}


In the health management system, management personnel can easily and accurately find and maintain relevant data through the system. The main data maintained by the system includes: manager identity information, main engine configuration information, experimental information, working condition information, and raw result file information. Therefore, a database table needs to be established for the above information.

After completing the structural design of the database, it is necessary to correlate the relationships between the database tables. The database tables do not exist independently. For example, each experiment contains multiple working conditions. Then the experimental and working conditions information is a oneto-many relationship. To ensure the consistency of the data, the relationship of the database needs to be further designed. The completed database diagram is shown in Fig. 4.

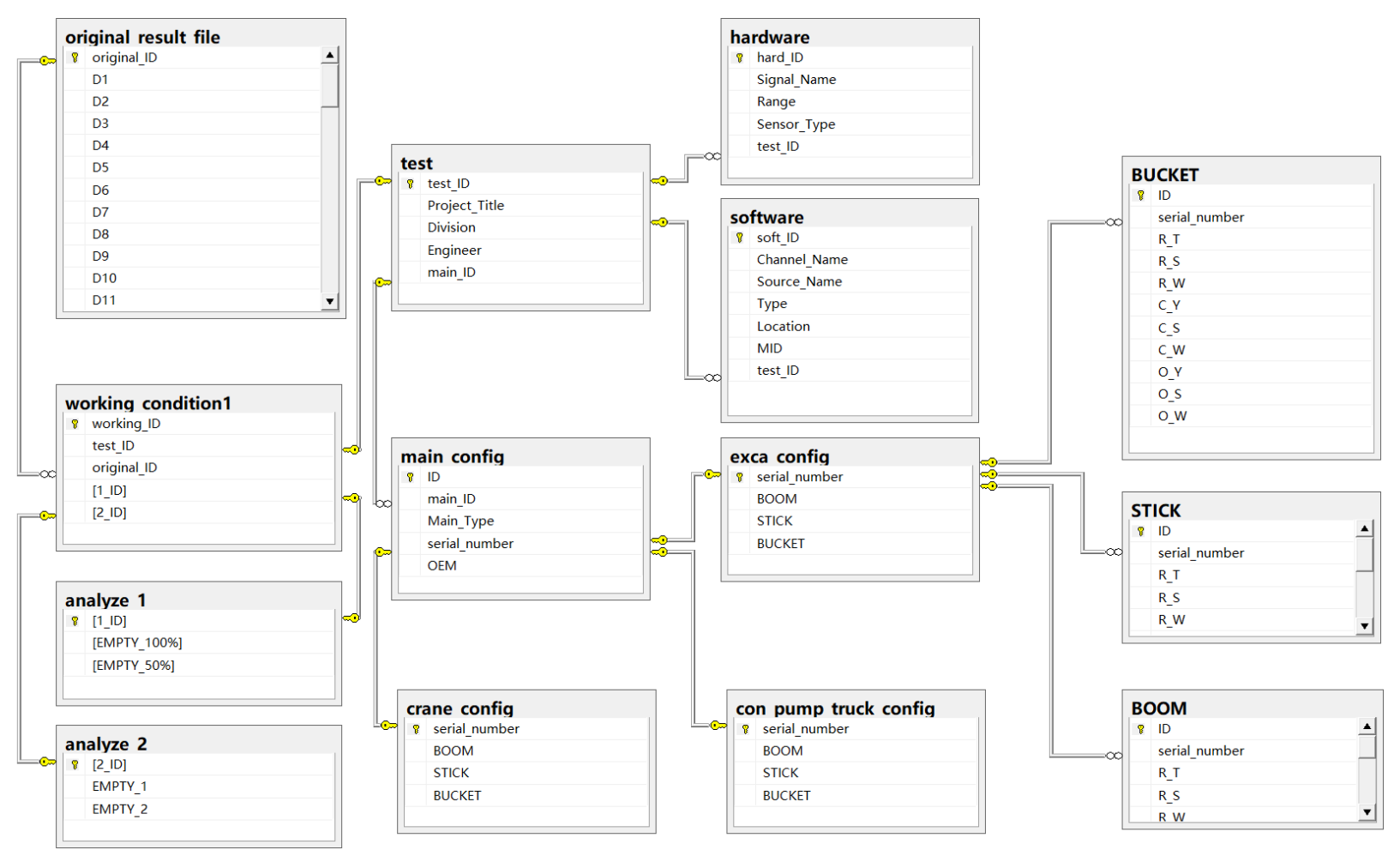

Fig. 4: Database diagram.

The database connection is the basis for the realization of the entire system function. Basically, all functions need to call the relevant data in the database. Therefore, before the realization of each function, you need to complete the relevant configuration of the database connection. The system uses the ADO.NET technology to connect to the database. First you need to define the database connection information in the Web.Config file. Including the server address, database name, account password, etc.

The definition of database connection class DbHelperSQL.cs, user-defined correlation method to execute SQL statements in this category. In the original reference should contain the System.Configuration namespace, and use PubConstant.cs to read Web.Config in the database connection string.

\section{System Function Implementation}

\subsection{Software Development Method}

The system is an open architecture. In the development mode, the software development model of the autonomous health management system adopts the software engineering idea. First, it puts forward a detailed user requirement for the functional requirements of health management, and then according to the software engineering method, each function is implemented. The demand analysis of the module is converted into the corresponding small module, and finally the componentized technology is used to implement each small module. The advantage of this solution is that the software system developed with this solution has very good reliability, maintainability, and testability, which is very necessary for the high reliability of the fault 
diagnosis system itself. In addition, different failure modes for the control system may not strictly distinguish its failure prediction and diagnosis methods, and each diagnosis method is provided in the form of a component. So that the scale of the system will be greatly reduced and the system efficiency will be improved. At the same time, the reproducibility of this health management system is very good, which will greatly reduce costs for the redevelopment of similar health management systems.

\subsection{VS Method to Call MATLAB Engine}

The MATLAB engine is a set of API function interfaces that users can use to call MATLAB functions by sending script commands. In a specific example, a $\mathrm{C} / \mathrm{C}++$ program acts as a front end and can send control commands to the MATLAB engine as a background. The MATLAB engine makes different responses depending on the type of control command. The essence of this method is that the ActiveX control calls the MATLAB process. DLL files, which are dynamic link library files, are part of the application. When the exe program starts, the corresponding DLL file will be called. MATLAB comes with a compiler. Users can use this compiler to compile already-written *.m files into DLL files, and then use MATLAB functions written directly in DLL files in $\mathrm{C} / \mathrm{C}++$ programs.

The algorithm of the condition identification and fault diagnosis module in this article are all written in MATLAB. Therefore, the method of using DLL to call MATLAB is used to call the algorithm in MATLAB. The matlab function is packaged into a DLL file, and the matlab data is combined to support the DLL (MWArray.dll) and delivered to the C\# program.

\subsection{Specific Function Implementation}

(1) Condition identification module: to realize data collection, process data into the database, and programmatically query main engine operating condition data. With the help of the condition identification model, the main engine condition is determined and the human-machine interaction interface displays the evaluation results.

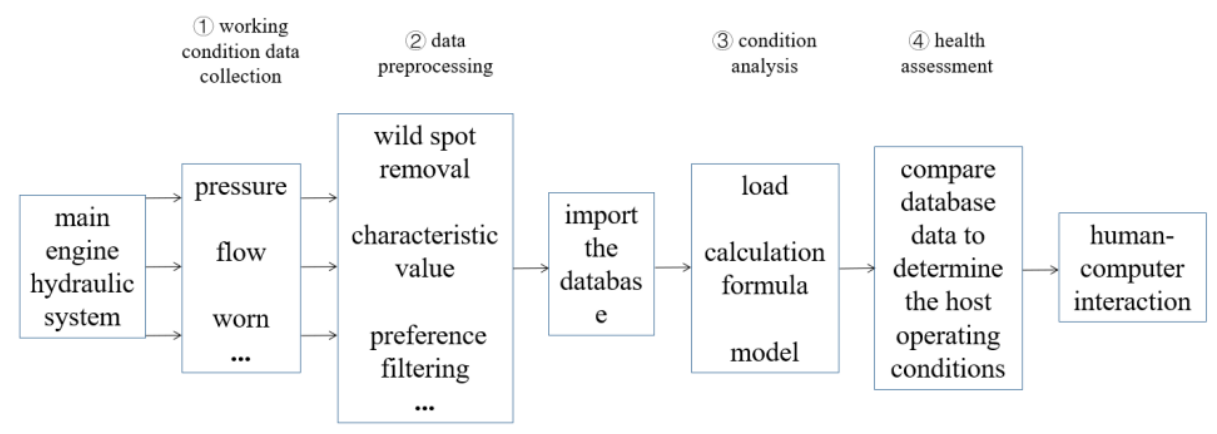

Fig. 5: Condition identification module flow chart.

(2) Energy consumption analysis module: Collect the data of the complete machine and the engine, and determine the match between power and gear through the energy consumption analysis model to evaluate the main engine health status.

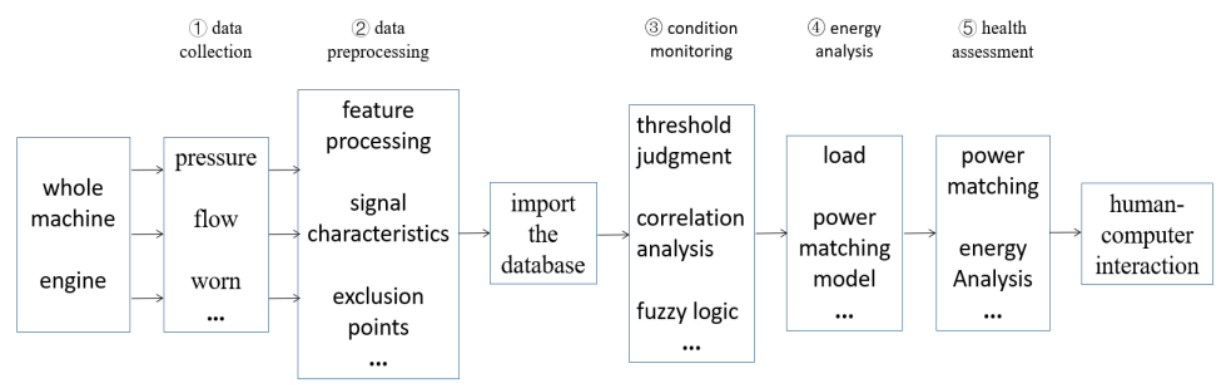

Fig. 6: Energy consumption analysis module flow chart. 
(3) Fault diagnosis module: detecting faults or anomalies, identifying the nature and severity of the faults, ranking the faults according to their importance and urgency, applying certain algorithm logic, comprehensively applying maintenance manuals, and repairing historical cases and other information to achieve The rapid diagnosis of hydraulic faults, and give a reasonable troubleshooting program.

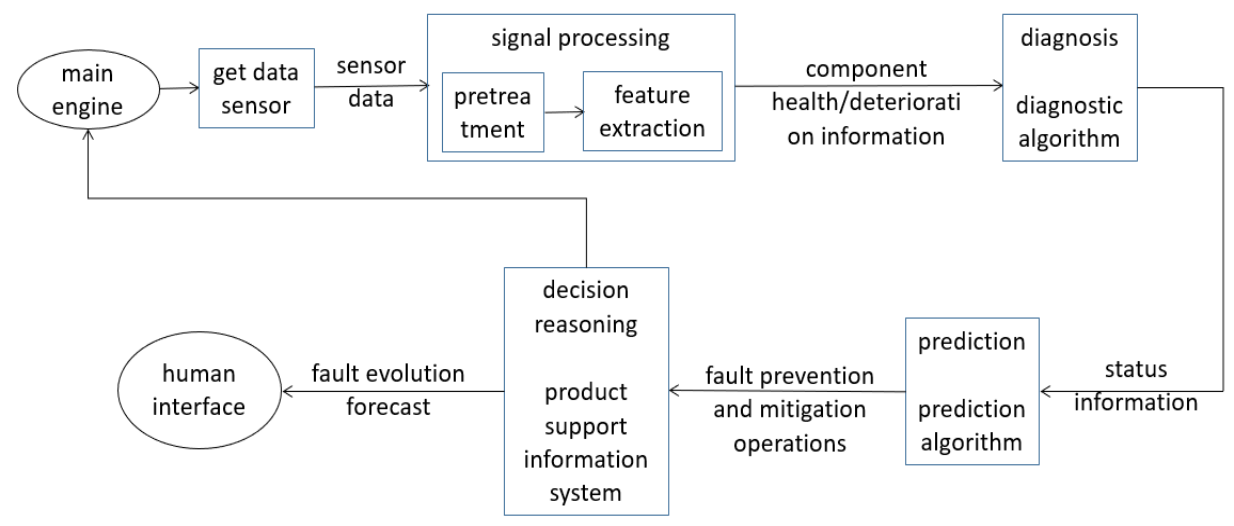

Fig. 7: Troubleshooting module flow chart.

\section{Conclusion}

This paper designs a small excavator health management system based on functional modules. The feasibility of the TBM tool information management system is discussed from system architecture, system function module design and database design. Finally, the designed system functions are implemented. The main conclusions are:

(1) Modular design according to function has certain reference effect on the design of future excavator health management system in China.

(2) By studying the hydraulic data, a working condition identification algorithm model and a fault diagnosis algorithm model were established to achieve adaptive component control and main engine operating conditions self-diagnosis.

(3) Using Matlab's powerful mathematics computing capabilities and drawing capabilities, VisualC\# calls the Matlab functions, making it easier to develop engineering applications.

(4) Further improvements can be made to the algorithms that implement the system functions so that it can monitor host health in real time.

\section{Acknowledgements}

This paper is supported by the Science and Technology Project of Strategic Emerging Industry in Hunan (Granted No. 2016GK4009) and the National High Technology Research and Development Program of China (Granted No. 2013AA040203).

\section{References}

[1] Zhang Renjing. Analysis of WY22 excavator working device based on ANSYS and Pro E[D]. Northeastern University, 2008.

[2] Zhang Tianrui. Research on Lifecycle Health Management Technology of Service-oriented Full-face Tunnelling Machines[D]. Shenyang: Northeastern University, 2014.

[3] Dong Zhicun. Research on Spacecraft Integrated Health Management System[D]. Harbin: Harbin Institute of Technology, 2013.

[4] Byington C S, Kalgren P W, Donovan B P, et al. Streamlined avionics PHM utilizing portable information and reasoning, Proceeding of Aerospace Conference. IEEE, 2005: 3547-3554.

[5] Li Linqi. Equipment health management system based on internet of things[D]. Xi'an: Xi'an University of Technology, 2013. 
[6] Staszewski W. Monitoring on-line integrated technologies for operational reliability--monitor. Air \& Space Europe, 2000, 2(4): 67-72.

[7] Zhang Baozhen. Development and Application of Prediction and Health Management Technology[J]. Mesures and Control Technology, 2008, 27(2): 5-7. 